\title{
FORMULATION OPTIMIZATION AND CHARACTERIZATION OF GANCICLOVIR LOADED DRY CHITOSAN NANOPARTICLES
}

\author{
RUTU PATEL ${ }^{1}$, GAYATRI PATEL ${ }^{1 *}$, BALARAM GAJRA ${ }^{1,2}$, RAJESH PARIKH ${ }^{1}$ \\ ${ }^{1}$ Department of Pharmaceutics and Pharmaceutical Technology, Ramanbhai Patel College of Pharmacy, Charotar University of Science \& \\ Technology, CHARUSAT Campus, Changa - 388 421, Gujarat, India. ${ }^{2}$ Walmart Pharmacy, 3600 Majormeckanzy Drive, Vaughan, Ontario, \\ Canada. Email: gayatripatel.ph@charusat.ac.in
}

Received: 17 November 2016, Revised and Accepted: 17 December 2016

ABSTRACT

Objective: The objective of this work was to formulate, optimize, and characterize ganciclovir (GCV) loaded dry chitosan nanoparticles (CSNPs).

Methods: The GCV loaded CSNPs was prepared by ionic gelation method. Box-Behnken design was employed to optimize the influence of independent process and formulation variables like drug to polymer ratio, concentration of sodium tripolyphosphate, and stirring time (min) on the dependent variables such as particle size (PS) and drug encapsulation efficiency (\% EE). The optimum conditions were determined by regression analysis of the output data.

Results: The independent variables had interactive effects and they affected both the responses. The optimum formulation had PS within the range of 100-120 nm and \% EE between 85\% and 86\%. The prepared GCV loaded CSNPs were dried by fluidized bed drying method. Fourier transform infrared spectra showed there was no physicochemical interaction between GCV and CS. Powder X-ray diffraction study showed less intense crystalline peaks indicated that GCV may exist in the formulation as amorphous nanodispersion or molecular dispersion form. Differential scanning calorimetry study was performed which indicated that the drug was molecularly dispersed inside the matrix of CS. Higuchi model was the best to fit the in vitro release data for the GCV loaded CSNPs.

Conclusion: From the results, it can be concluded that the GCV loaded dry CSNPs were formulated, optimized, and characterized using desired pharmacotechnical properties.

Keywords: Chitosan nanoparticles, Box-Behnken design, Sodium tripolyphosphate, Ionic gelation.

(C) 2017 The Authors. Published by Innovare Academic Sciences Pvt Ltd. This is an open access article under the CC BY license (http://creativecommons. org/licenses/by/4. 0/) DOI: http://dx.doi.org/10.22159/ajpcr.2017.v10i3.16205

\section{INTRODUCTION}

Polymeric nanoparticles (NPs) have been studied as promising drug delivery systems. Different NPs have been formulated and characterized with the use of natural as well as synthetic polymers. Based on the physicochemical and biological properties, chitosan (CS) a natural polymer attracts the researchers to work with Hamidi et al. [1].

CS has been proved to have many other intrinsic properties, such as low toxicity (lethal dose $50 \%$ in mice is $16 \mathrm{~g} / \mathrm{kg}$ body weight) [2], biocompatibility and biodegradability (Cordova et al., 2008). It is mucoadhesive polymer and can increase the residence time at the site of absorption and has favorable controlled drug-release abilities [3]. It has been demonstrated that CS (when protonated) affects cell permeability and enhances paracellular permeability of drugs across the mucosal epithelia by opening the intercellular tight junctions.

Ganciclovir (GCV) 9-(1,3-dihydroxy-2-propoxymethyl) guanine, is a Biopharmaceutics Classification System Class III drug. GCV ( $\log p=-1.7$ ) having molecular weight $255.2 \mathrm{~g} / \mathrm{mol}$. It is the first antiviral drug that proved to be efficacious in the treatment of Cytomegalovirus (CMV) disease in humans [4]. GCV is also used for maintenance therapy and prophylaxis of CMV. Oral bioavailability of GCV is $\sim 5 \%$. Possible reasons for the poor bioavailability are poor permeability through the gastrointestinal tract (GIT) and P-glycoprotein substrate activity [5]. Therefore, it is necessary to develop a drug delivery system for GCV which can enhance the absorption of GCV. Different drug delivery systems for GCV have been developed these days, such as GCV loaded albumin NPs [6], solid lipid NPs [7], Long-circulating liposome-encapsulated GCV [8].
This study focus on development of GCV loaded dry CSNPs with an ionic gelation method. The $2^{5-2}$ fractional factorial design was employed to screen the process and formulation parameters. Response surface methodology with the utilization of Box-Behnken experimental design (BBD) was employed for optimization of the particle size (PS) and entrapment efficiency (\% EE) of GCV loaded dry CSNPs, as they are properties of great influence on the pharmacokinetic (i.e., biodistribution) and the pharmacodynamic (i.e., therapeutic efficacy) of the drug loaded NPs [9]. The optimized formulation was investigated for physicochemical characterizations such as scanning electron microscopy (SEM), Fourier transform infrared spectroscopy (FTIR), and differential scanning calorimetry (DSC) study were employed for detection of particle morphology and any physicochemical interaction with the CS. In vitro release profile was investigated.

\section{MATERIALS AND METHODS}

\section{Materials}

GCV was kindly gifted by Bakul Fine Chem Research Center, Mumbai. CS (molecular weight=110 kDa, $80.0 \%$ degree of deacetylation) was gratis sample from Chitopharm S, Norway (USA). Sodium tripolyphosphate (TPP) (Cross-linking agent) was purchased from Sigma-Aldrich, Mumbai (India). The water used was pretreated with the Milli-Q plus system (Millipore, Q-5 UVS, India). All other materials of analytical grade were used.

\section{Methods}

Preparation of GCV loaded CSNPS

The GCV loaded CSNPs were prepared by ionic gelation method [10]. The possible mechanism for ionic gelation is that the ionic interaction 
between positively charged CS with negatively charged sodium TPP [11]. Briefly, CS solutions were prepared by dissolving CS into Milli- $Q^{\circledR}$ water at different concentrations containing glacial acetic acid $(2 \%)$. GCV was dissolved in Milli- $Q^{\circledR}$ water containing different concentrations of TPP. NPs were formed by adding TPP solution drop wise onto a CS solution under mechanical stirring (Remi, India) at room temperature. Thereafter, the CS-TPP particle suspension was processed under ultrasonication by Probe sonicator (VCX-500, Vibra cell, USA) for 5 minutes, producing CSNPs with controlled PS. The prepared GCV loaded CSNPs were dried by fluidized bed drying (FBD) method [12]. The GCV loaded CSNPs dispersion was centrifuged at $10,000 \mathrm{rpm}$ for 30 minutes. The sediment was mixed with microcrystalline cellulose and Aeroperl R 300 in the ratio of 3:1. The prepared wet mass was subjected to FBD at $60-70^{\circ} \mathrm{C}$ for $4-6$ minutes. The dried particles were allowed to pass from sieve $40 \#$ and further characterized.

\section{Experimental design}

From the prior experience and literature survey, the process and formulation parameters were identified and screened through $2^{5-2}$ fractional factorial design. Eight batches were prepared and evaluated for PS, \% EE and morphology. Drug:polymer ratio (w/w), concentration of TPP (\%w/v), stirring time (min), sonication time (min), and stirring speed $(\mathrm{rpm})$ were taken as the independent variables while PS (nm), EE (\%), and morphology were taken as dependent variables for $2^{5-2}$ fractional factorial design.

A BBD was used with, three-factors, three-level, and fifteen-runs to optimize the preparation conditions. The polynomial equations relating factors and responses were obtained by design of experiment software ${ }^{\circledR}$ (9.0.6). Table 1 shows the three variables (Drug:polymer ratio, concentration of TPP, and stirring time) were represented by $\mathrm{A}, \mathrm{B}$, and $\mathrm{C}$, respectively. The responses (PS and \%EE) were represented by $Y_{1}$ and $Y_{2}$, respectively [13]. A number of check-point experiments were carried out in the optimal area of the BBD to verify the optimization procedure validation, as predicted by desirability analysis and by comparing the experimental and predicted response.

\section{Characterization}

PS determination

The size of the particles was determined by dynamic laser scattering technique (Malvern nano S90, Malvern Instruments, UK) PS analyzer at temperature of $25^{\circ} \mathrm{C}$ with an angle of 900 .

\section{Entrapment efficiency (\% EE)}

$\%$ EE of the drug was determined by high-performance liquid chromatography (HPLC) (LC-2010C HT, Shimadzu, Japan). Formulations were centrifuged at 10,000 RPM for 30 minutes. Supernant was collected and analyzed for drug content at $254 \mathrm{~nm}$ by HPLC (LC-2010C HT, Shimadzu, Japan) [14]. The \% EE was calculated as follows:

$\% \mathrm{EE}=(\mathrm{Sa}-\mathrm{Sb}) / \mathrm{Sa}^{*} 100$

Where, Sa is the total amount of drug in system, Sb is the amount of drug in supernant after centrifugation.

Table 1: Variables with their coded and actual values for BBD

\begin{tabular}{llll}
\hline Independent variables & Low & Medium & High \\
\hline Coded values & $(-1)$ & $(0)$ & $(1)$ \\
A=Drug: Polymer ratio (w:w) & $1: 2$ & $1: 3$ & $1: 4$ \\
B=Concentration of TPP $(\% \mathrm{w} / \mathrm{v})$ & 0.3 & 0.4 & 0.5 \\
C=Stirring time (min) & 50 & 100 & 150 \\
\hline
\end{tabular}

Dependent variables constrains

$\mathrm{Y}_{1}=$ Particle size $(\mathrm{nm}) 100-200 \mathrm{~nm}$

$\mathrm{Y}_{2}=\mathrm{EE}(\%)$ Maximum

EE: Entrapment efficiency, BBD: Box-Behnken experimental design

\section{Morphology observation}

SEM (JSM 6010 LA, JEOL, USA) was used to access the morphology/shape of the dried CSNPs. A sample of dried CSNPs was placed on a double stick tape over aluminum stubs to get a uniform layer of particles. Sample was platinum coated for 20 seconds. Then the sample was observed by SEM at $10 \mathrm{kV}$ [15].

\section{FTIR spectroscopy}

FTIR spectrum was recorded using NICOLET-6700 (Thermo Scientific, USA) to confirm the cross-linking reaction between the phosphoric group of the TPP and amino group of CS. The pellet was prepared by homogenously dried formulation in dried potassium bromide in a mortar and pestle and then powder was compressed under vacuum using round flat face punch to produce pellet compact. The sample was placed in IR light path and spectra were scanned over the wavelength number range of 4000-400 $\mathrm{cm}^{-1}$ [16].

\section{DSC study}

DSC study was performed by DSC (DSC-60, Shimadzu Corporation, Japan) to characterize the physical state of GCV in CSNPs [17]. The heat flow as a function of temperature was measured over a temperature range of $50-400^{\circ} \mathrm{C}$ at heating rate of $10^{\circ} \mathrm{C} / \mathrm{min}[18]$.

\section{In vitro drug release study}

In vitro drug release study of GCV loaded CSNPs dispersion, marketed formulation (Natclovir: $250 \mathrm{mg}$ Capsule) and drug solution was carried out in diffusion cell apparatus (J-FDC-07, Orchid Scientifics and Innovations India Pvt., Ltd.) in phosphate buffer $\mathrm{pH}$ 6.8. At predetermined time intervals, the samples were withdrawn and replenish with fresh medium and the absorbance was measured by HPLC at $254 \mathrm{~nm}$. Data obtained from the in vitro drug release for formulation were fitted to various kinetic models. Each experiment was performed in triplicate. The drug release mechanism and linearization were determined by finding the goodness of fit $\left(R^{2}\right)$ and sum squared of residuals (SSR) for each kinetic model [19].

\section{RESULTS AND DISCUSSIONS}

The $2^{5-2}$ fractional factorial design revealed that the drug to polymer ratio, concentration of TPP, and stirring time was affected more significantly on the PS and \% EE. The PS was found to be in the range of $112.6 \pm 1.1$ to $364.9 \pm 4.759 \mathrm{~nm}$ and $\% \mathrm{EE}$ in the range of $10 \pm 5.4$ to $83.57 \pm 1.4 \%$ with spherical shape.

\section{Model fitting by BBD}

The screening design suggested that drug to polymer ratio, concentration of TPP, and stirring time were most significant factors. These significant factors were further optimized through the BBD. The measured significant variables range between $121.20 \pm 2.7$ and $294.5 \pm 15.4 \mathrm{~nm}$ for PS and between $59.95 \pm 0.5$ and $85.15 \pm 1.1$ for $\%$ EE. These indicated that PS and \% EE of the prepared CSNPs were largely influenced by the selected variables. The polynomial equations for both responses were as follows:

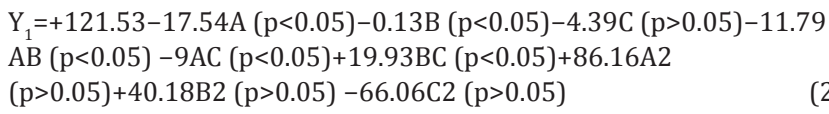

$\mathrm{Y}_{2}=+84.02-0.47 \mathrm{~A}(\mathrm{p}<0.05)+2.55 \mathrm{~B}(\mathrm{p}<0.05)-0.53 \mathrm{C}(\mathrm{p}<0.05)$

$+3.58 \mathrm{AB}(\mathrm{p}<0.05)-3.23 \mathrm{AC}(\mathrm{p}<0.05)-2.78 \mathrm{BC}(\mathrm{p}>0.05)-12.11 \mathrm{~A} 2$

$(p>0.05)-5.98 B 2(p>0.05)+0.085 C 2(p>0.05)$

Where, $\mathrm{Y}_{1}$ is the $\mathrm{PS}, \mathrm{Y}_{2}$ is the \% EE. A, B, and C is drug:polymer ratio, concentration of TPP and stirring time, respectively. $\mathrm{AB}, \mathrm{AC}$, and $\mathrm{BC}$ are the interaction terms which show how the responses change when two variables are concurrently changed, while the effect of changes in each single variable on the response is reflected by $\mathrm{A}, \mathrm{B}$, and $\mathrm{C}$ the main effect terms and $\mathrm{A}^{2}, \mathrm{~B}^{2}$, and $\mathrm{C}^{2}$ the quadratic terms. Table 2 presents the results of which the analysis of variance (ANOVA) was conducted to test the 
quadratic models significance and the lack of fit for the experimental data.

Equations (2) and (3) show the effect of the independent variables on the PS and \% EE. As A, B, and C the three independent variables changed simultaneously, the coefficient of interaction of $Y_{1}$ and $Y_{2}$ also changed. There is increase in the PS due to increase in the concentration of TPP would be due to cross-linkage between TPP and CS. As there is increase in the concentration of TPP, there would be more tripolyphosphoric ions to cross-link with amino groups on CS chains. As stirring time increases, there is increase in the PS. This may be due to aggregation of particles [20].

There is decrease in the \% EE with increase in the drug:polymer ratio. This may be due to the fact that at higher concentration CS molecules in the dispersion are present very close to each other during precipitation by polyanion. Hence, they got precipitated to form a large particle and this larger particle is having low entrapment of drug [20].

To check the significance of the quadratic models and the lack of fit for the experimental data the ANOVA was conducted (Table 2). Fischer's ratio ( $F$ ) confirms the models significances, where $\mathrm{FY}_{1}=9.71$ $(\mathrm{p}=0.011<0.05)$ and $\mathrm{FY}_{2}=5.93(\mathrm{p}=0.032<0.05)$. The value of correlation coefficient $\left(R^{2}\right)$ for equation was found to be $R_{1}{ }^{2}=0.961$ and $R_{2}^{2}=0.934$, indicating a good fit.

The Student's t-test was applied to determine the significant difference between actual and predicted value of dependent parameters. The p-value for the PS and \% EE was found to be 0.372 and 0.468 , respectively, which showed that at $5 \%$ level of significance, there is no significant difference between actual and predicted values of PS and \% EE.

Fig. 1 shows the three-dimensional response surface plots of independent variables interactive effects on the PS and \% EE. Optimum

Table 2: ANOVA analysis for measured responses

\begin{tabular}{lllllll}
\hline Response & Source & $\mathbf{d f}^{\mathbf{a}}$ & $\mathbf{S S}^{\mathbf{b}}$ & $\mathbf{M S}^{\mathbf{c}}$ & F ratio $^{\mathbf{d}}$ & $\mathbf{p ~ v a l u e ~}$ \\
\hline $\mathrm{Y}_{1}$ & Model & 9 & 48816.51 & 5424.06 & 9.71 & 0.111 \\
& Error & 5 & 2794.25 & 558.85 & - & - \\
$\mathrm{Y}_{2}$ & Total & 14 & 51610.76 & - & - & - \\
& Model & 9 & 823.00 & 91.44 & 5.93 & 0.0321 \\
& Error & 5 & 77.10 & 15.42 & - & - \\
& Total & 14 & 900.10 & - & - & - \\
\hline
\end{tabular}

${ }^{a}$ Degree of freedom, ${ }^{b}$ Sum of square, ${ }^{\mathrm{c}}$ Mean sum of square, ${ }^{\mathrm{d}}$ Model MS/error MS, ANOVA: Analysis of variance conditions for preparation of CSNPs can be achieved for different values of independent variables within the experimental range, followed by desirability function. At optimized conditions, GCV loaded CSNPs had the PS $121.20 \pm 2.7 \mathrm{~nm}$ and \% EE of $85.15 \pm 1.1 \%$ with the desirability of 0.978 (Fig. 2). There was no significant difference between the predicted and actual values of the measured responses as the $p>0.05$. Fig. 3 shows overlay contour plot of the location of the desirable region for selection of optimized NP formulation of GCV.

\section{Morphology observation}

SEM micrographs are shown in Fig. 4 which revealed that prepared particles were spherical in shape which is favorable for the uptake through the GIT.

\section{FTIR study}

In the FTIR spectra of cross-linked CS (Fig. 5), the peak of $1655 \mathrm{~cm}^{-1}$ disappeared and two new peaks at $1645 \mathrm{~cm}^{-1}$ and $1554 \mathrm{~cm}^{-1}$ appeared. The disappearance of the band could be attributed to the linkage between the phosphoric and ammonium ions. The cross-linked CS also showed a peak for $\mathrm{P}=0$ at $1155 \mathrm{~cm}^{-1}$ [21]. Cross-linked CS-TPP in the NPs also shows broader peak at 2934-3421 $\mathrm{cm}^{-1}$ indicates hydrogen bonding between $\mathrm{OH}$ and $\mathrm{NH}_{2}$ groups [22]. Hence, this confirms the cross-linking between CS and TPP.

\section{DSC study}

DSC studies (Fig. 6) were performed to understand the behavior of the cross-linked CS on application of thermal energy. Polysaccharides usually have a strong affinity for water, and in solid state, these macromolecules may have disordered structures that can be easily hydrated. The endotherm related to evaporation of water is expected to reflect the molecular changes brought in after cross-linking.

The thermogram of $\mathrm{CS}$ showed endotherm at $102.36^{\circ} \mathrm{C}$ with the enthalpy of fusion $(\Delta \mathrm{H}) 81.93 \mathrm{~J} / \mathrm{g}$. The water-holding capacity of crosslinked CS with TPP was found to be more $(\Delta \mathrm{H} 244.24 \mathrm{~J} / \mathrm{g})$ compared with that of plain CS. The cross-linking of CS with TPP at different $\mathrm{pH}$ modifies the crystalline nature of CS. The hydrophilicity of cross-linked $\mathrm{CS}$ is higher at $\mathrm{pH} 3$, which might be responsible for its increase in the water holding capacity. On the basis of these results, it can be stated that increase in the polar groups and reduction in crystalline domains caused an increase in the water-holding capacity of cross-linked CS. These results are in agreement with the work done by Kittur [23]. An endothermic melting peak of GCV was observed at $253.88^{\circ} \mathrm{C}$. The absence of this peak in the thermogram of formulation indicates that the drug was molecularly dispersed inside the matrix of CS as a solid solution.
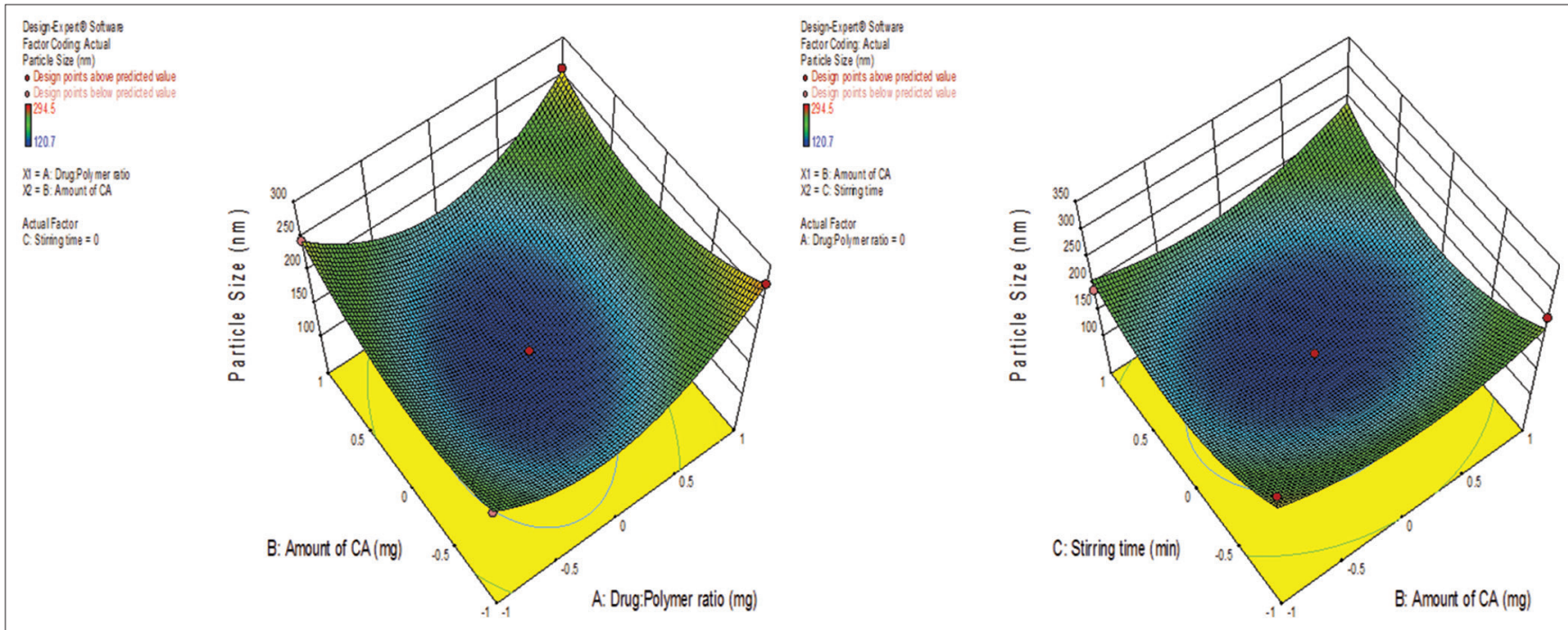

Fig. 1: Three-dimensional response surface curves for particle size 


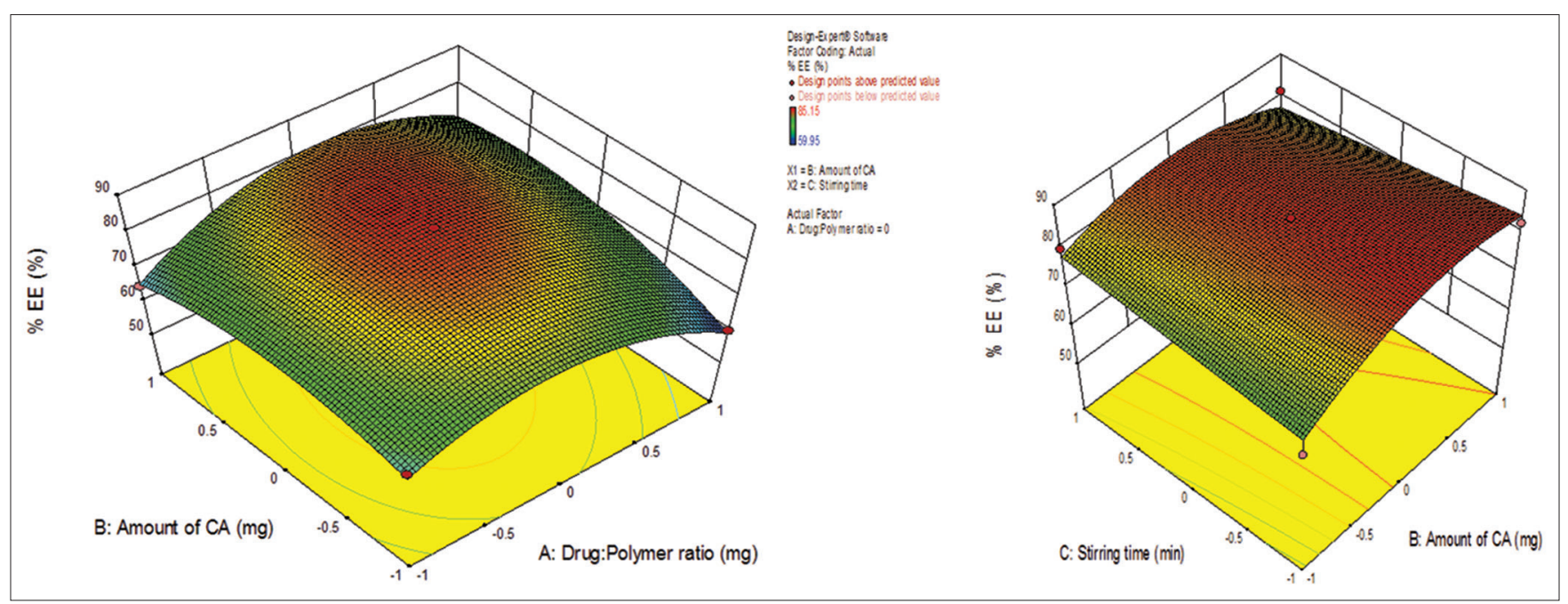

Fig. 2: Three-dimensional response surface curves for $\%$ entrapment efficiency

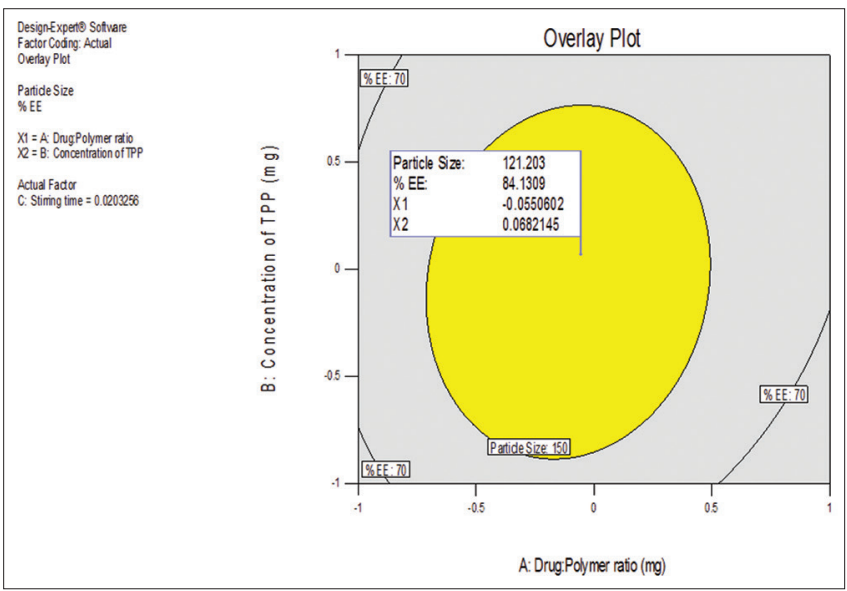

Fig. 3: Overlay contour plot showing the location of the desirable region for selection of optimized nanoparticle formulation of ganciclovir

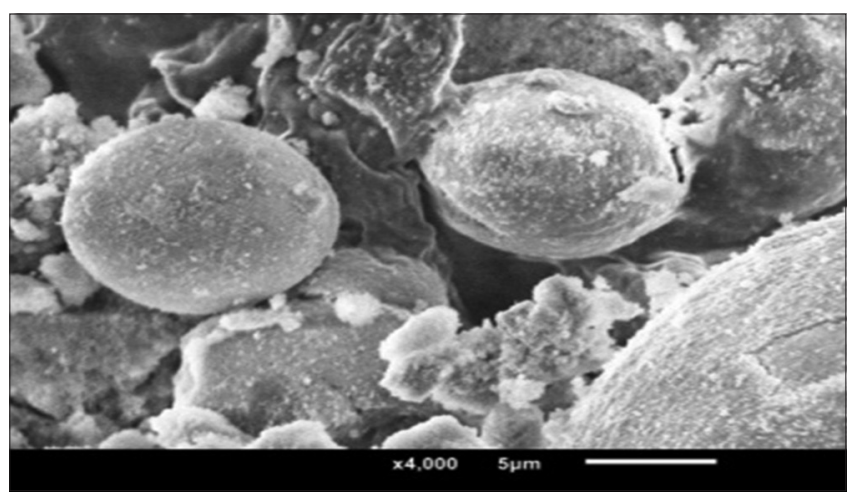

Fig. 4: Scanning electron microscopy micrograph of ganciclovir loaded chitosan nanoparticles

\section{In vitro drug release study}

The in vitro drug release study of GCV loaded CSNPs, marketed formulation and drug solution was carried out in $\mathrm{pH} 6.8$ phosphate buffer. Drug exhibited sustained release behavior up to the $24 \mathrm{hrs}$. Drug release was found up to $71.63 \pm 0.36 \%, 45.36 \pm 1.96 \%$, and $27.23 \pm 1.19 \%$ in GCV loaded CSNPs, marketed formulation and drug solution, respectively, at the end of $24 \mathrm{hrs}$. It was noteworthy that

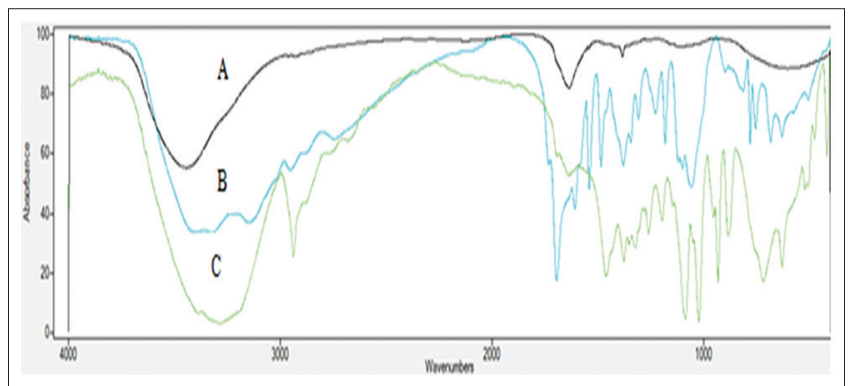

Fig. 5: Fourier transform infrared spectra of (a) Chitosan, (b) ganciclovir (GCV), (c) GCV loaded chitosan nanoparticles

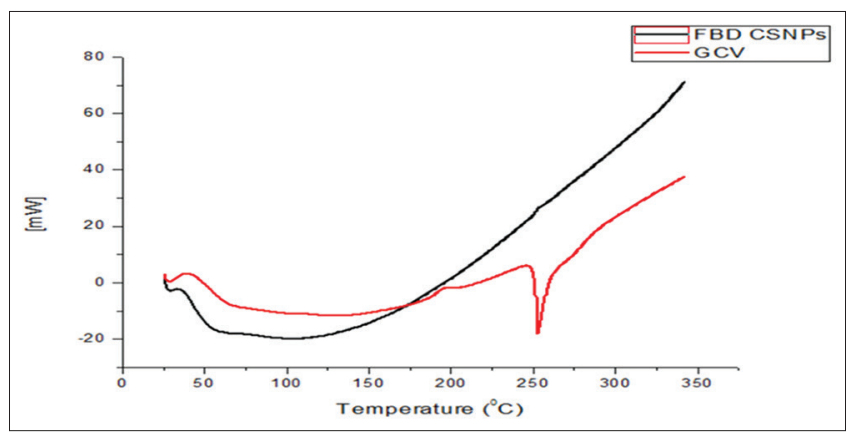

Fig. 6: The overlay differential scanning calorimetry thermogram of ganciclovir (GCV) and GCV loaded chitosan nanoparticles (CSNPs) (fluidized bed drying CSNPs)

formulation showed an initial burst release possibly due to the small size of the NPs. As the particle diameter was reduced, the specific surface area increased, while the path length to the surface of the drug decreased [24]. In the comparison with the marketed formulation the prepared GCV loaded CSNPs released drug in higher amount. The model having highest value of $\mathrm{R}^{2}$ and lowest value of SSR is considered as the best model to fit [25]. From the results, it was found that drug release from CSNPs follows Higuchi model $\left(\mathrm{R}^{2}=0.9078\right.$; $\left.\mathrm{SSR}=0.3796\right)$ indicating that drug release as a diffusion process based on the Fick's law [18].

\section{CONCLUSION}

In the presented research, the process and formulation parameters were optimized through the BBD. The variables drug to polymer 
ratio, concentration of TPP, and stirring time affected the PS and \% EE more significantly. The GCV loaded dry CSNPs showed diffusion controlled release mechanism up to $24 \mathrm{hrs}$ along with ease of formulation.

\section{ACKNOWLEDGMENTS}

The author is thankful to Dr. R. H. Parikh for his scientific advice. The author wants to thank Ms. Rutu Patel for her writing assistance. The Authors Acknowledge Ramanbhai Patel College of Pharmacy, Charotar University of Science and Technology (CHARUSAT) for financial support.

\section{REFERENCES}

1. Hamidi M, Azadi A, Rafiei P. Hydrogel nanoparticles in drug delivery. Adv Drug Deliv Rev 2008;60(15):1638-49.

2. Agnihotri SA, Mallikarjuna NN, Aminabhavi TM. Recent advances on chitosan-based micro-and nanoparticles in drug delivery. J Control Release 2004;100(1):5-28.

3. Wang XY, Jiang XP, Li Y, Zeng S, Zhang YW. Preparation Fe3O4@ chitosan magnetic particles for covalent immobilization of lipase from Thermomyces lanuginosus. Int J Biol Macromol 2015;75:44-50.

4. Anderson RD, Griffy KG, Jung D, Dorr A, Hulse JD, Smith RB. Ganciclovir absolute bioavailability and steady-state pharmacokinetics after oral administration of two $3000-\mathrm{mg} / \mathrm{d}$ dosing regimens in human immunodeficiency virus-and cytomegalovirus-seropositive patients. Clin Ther 1995;17(3):425-32.

5. Li M, Si L, Pan H, Rabba AK, Yan F, Qiu J, et al. Excipients enhance intestinal absorption of ganciclovir by P-gp inhibition: Assessed in vitro by everted gut sac and in situ by improved intestinal perfusion. Int J Pharm 2011:403(1-2):37-45.

6. Merodio M, Arnedo A, Renedo MJ, Irache JM. Ganciclovir-loaded albumin nanoparticles: Characterization and in vitro release properties. Eur J Pharm Sci 2001;12(3):251-9.

7. Ren J, Zou M, Gao P, Wang Y, Cheng G. Tissue distribution of borneolmodified ganciclovir-loaded solid lipid nanoparticles in mice after intravenous administration. Eur J Pharm Biopharm 2013;83(2):141-8.

8. Kajiwara E, Kawano K, Hattori Y, Fukushima M, Hayashi K, Maitani Y. Long-circulating liposome-encapsulated ganciclovir enhances the efficacy of HSV-TK suicide gene therapy. J Control Release 2007;120(1-2):104-10.

9. Georgieva D., Kostova B., Rachev D. Nanosized polymeric carriers for dexamethasone controlled delivery. J Chem 2015;7 Suppl 3:1767-72.

10. Ruby JJ, Pandey VP. Chitosan nanoparticles as a nasal drug delivery for memantine hydrochloride. Int J Pharm Pharm Sci 2014;7(1):34-7.
11. Calvo P, Remuñan-López C, Vila-Jato JL, Alonso MJ. Chitosan and chitosan/ethylene oxide-propylene oxide block copolymer nanoparticles as novel carriers for proteins and vaccines. Pharm Res 1997;14 Suppl 10:1431-6.

12. Egashira K. Pharmaceutical Composition Containing StatinEncapsulated Nanoparticle. Google Patents; 2007.

13. Bei D, Marszalek J, Youan BB. Formulation of dacarbazine-loaded cubosomes-part I: Influence of formulation variables. AAPS Pharm Sci Technol 2009;10(3):1032-9.

14. Elmowafy E, Osman R, El-Shamy AE, Awad GA. Stable colloidal chitosan/alginate nanocomplexes: Fabrication, formulation optimization and repaglinide loading. Int J Pharm Pharm Sci 2014;6(2):520-5.

15. Desai MP, Labhasetwar V, Amidon GL, Levy RJ. Gastrointestinal uptake of biodegradable microparticles: Effect of particle size. Pharm Res 1996;13(12):1838-45.

16. Ray M, Pal K, Anis A, Banthia AK. Development and characterization of chitosan-based polymeric hydrogel membranes. Des Monomers Polym 2010;13(3):193-206.

17. Sambandam B, Kumar S, Ayyaswamy A, Yadav BV, Thiyagarajan D. Synthesis and characterization of poly DL lactide (PLA) nanoparticles for the delivery of quercetin. Int J Pharm Pharm Sci 2015;7 Suppl 5:42-9.

18. Guhagarkar SA, Gaikwad RV, Samad A, Malshe VC, Devarajan PV. Polyethylene sebacate-doxorubicin nanoparticles for hepatic targeting. Int J Pharm 2010;401(1-2):113-22.

19. Dash S, Murthy PN, Nath L, Chowdhury P. Kinetic modeling on drug release from controlled drug delivery systems. Acta Pol Pharm 2010;67:217-23.

20. Dangi RS, Shakya S. Preparation, optimization and characterization of PLGA nanoparticle. Int J Pharm Life Sci 2013;4(7):2810-8.

21. Bhumkar DR, Pokharkar VB. Studies on effect of $\mathrm{pH}$ on cross-linking of chitosan with sodium tripolyphosphate: A technical note. AAPS Pharm Sci Technol 2006;7(2):E50.

22. Anbarasan B, Menon VV, Niranjana V, Sundara R. Optimization of the formulation and in-vitro evaluation of chloroquine loaded chitosan nanoparticles using ionic gelation method. J Chem Pharm Sci 2013;6 Suppl 1:66-72.

23. Tharanathan RN, Kittur FS. Chitin - the undisputed biomolecule of great potential. Crit Rev Food Sci Nutr 2003:43(1):61-87.

24. Cordova M, Cheng M, Trejo J, Johnson SJ, Willhite GP, Liang JT, et al. Delayed HPAM gelation via transient sequestration of chromium in polyelectrolyte complex nanoparticles. Macromolecules 2008;41 Suppl 12:4398-404

25. Singh J, Gupta S, Kaur H. Prediction of in vitro drug release mechanisms from extended release matrix tablets using SSR $/ \mathrm{R}^{2}$ technique. Trends Appl Sci Res 2011;6 Supp14:400-9. 